NASA Contractor Report 194979

ICASE Report No. 94-76
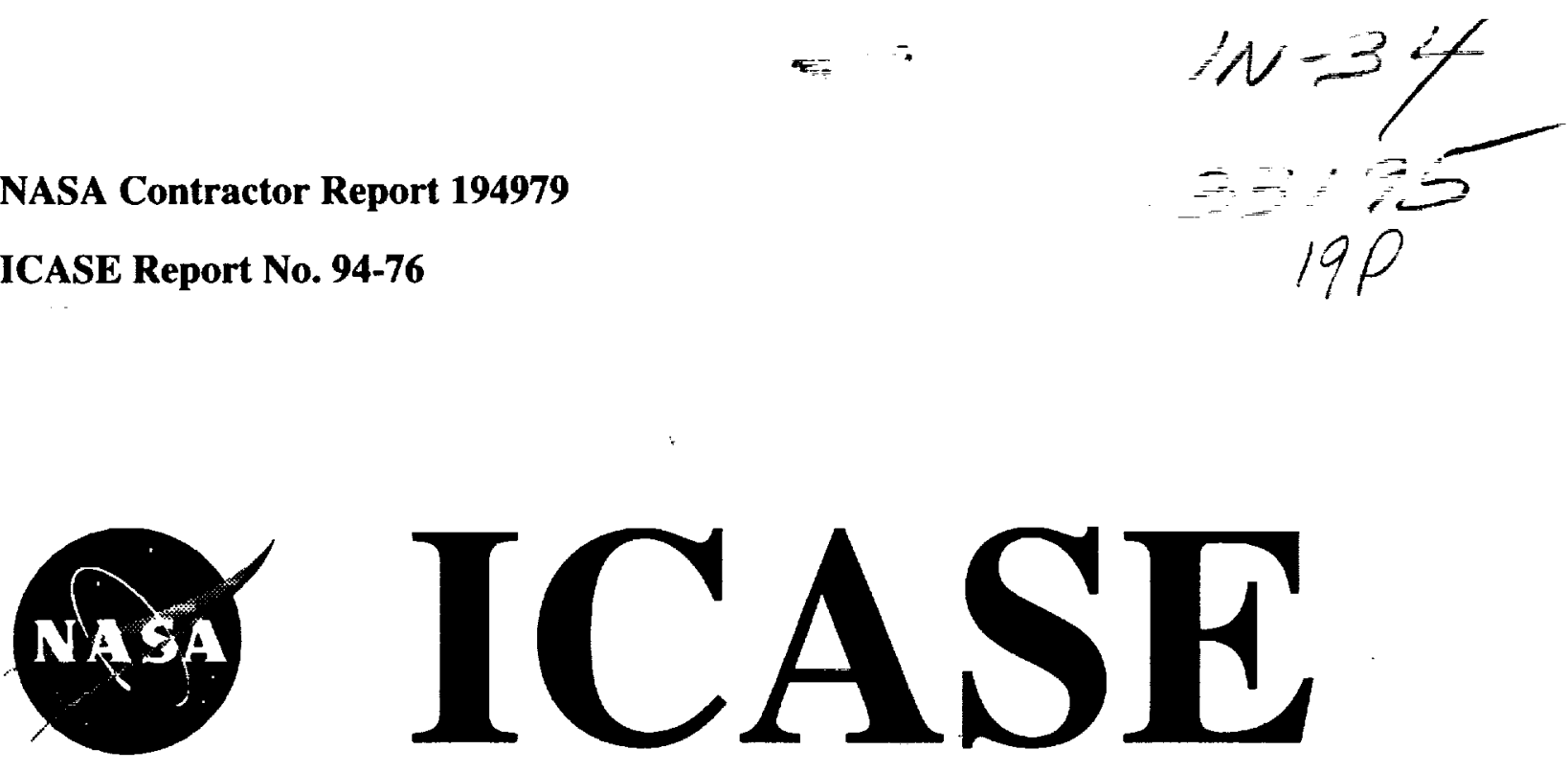

\title{
A MODIFIED RESTRICTED EULER EQUATION FOR TURBULENT FLOWS WITH MEAN VELOCITY GRADIENTS
}

Sharath S. Girimaji

Charles G. Spezia'n

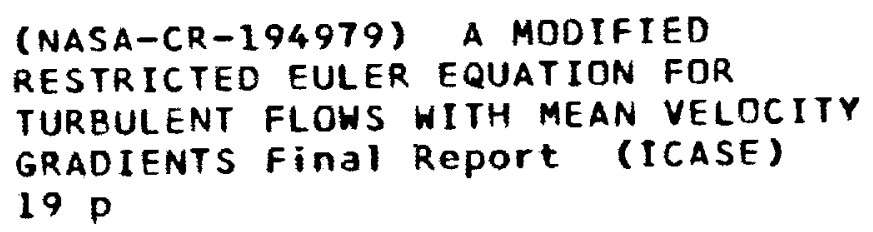

N95-16070

Unclas

G3/34 0033195

Contract NAS1-19480

September 1994

Institute for Computer Applications in Science and Engineering NASA Langley Research Center Hampton, VA 23681-0001

SRA Operated by Universities Space Research Association 


\title{
A MODIFIED RESTRICTED EULER EQUATION FOR TURBULENT FLOWS WITH MEAN VELOCITY GRADIENTS
}

\author{
Sharath S. Girimaji* \\ ICASE, NASA Langley Research Center \\ Hampton, VA 23681 \\ Charles G. Speziale* \\ Department of Aerospace and Mechanical Engineering \\ Boston University \\ Boston, MA 02215
}

\begin{abstract}
The restricted Euler equation captures many important features of the behavior of the velocity gradient tensor observed in direct numerical simulations (DNS) of isotropic turbulence. However, in slightly more complex flows the agreement is not good, especially in regions of low dissipation. In this paper, it is demonstrated that the Reynolds-averaged restricted Euler equation violates the balance of mean momentum for virtually all homogeneous turbulent flows with only two major exceptions: isotropic and homogeneously-sheared turbulence. A new model equation which overcomes this shortcoming and is more widely applicable is suggested. This model is derived from the Navier-Stokes equation with a restricted Euler type approximation made on the fluctuating velocity gradient field. Analytical solutions of the proposed modified restricted Euler equation appear to be difficult to obtain. Hence, a strategy for numerically calculating the velocity gradient tensor is developed. Preliminary calculations tend to indicate that the modified restricted Euler equation captures many important aspects of the behavior of the fluctuating velocity gradients in anisotropic homogeneous turbulence.
\end{abstract}

*This research was supported by the National Aeronautics and Space Administration under NASA Contract No. NAS1-19480 while the author was in residence at the Institute for Computer Applications in Science and Engineering (ICASE), NASA Langley Research Center, Hampton, VA 23681-0001. 
101 


\section{Introduction}

More detailed knowledge of the behavior of the velocity gradient tensor is crucial for a better understanding of turbulent flows (e.g., vortex stretching which affects the energy cascade). Recently, an examination of direct numerical simulation (DNS) data bases has revealed certain universalities in the geometry of the velocity gradient tensor in simple flows as well as in high dissipation regions of more complex flows (see Ashurst et al [1]). Two important features are frequently observed concerning the geometry of the velocity gradient tensor: (i) the intermediate eigenvalue of the strain rate tensor is often small in magnitude and positive in sign; and (ii) the vorticity vector is aligned along this intermediate eigenvector. A simple dynamical model derived from the Euler equation - commonly called the restricted Euler equation - reproduces this apparently universal behavior of the velocity gradients. The restricted Euler equation was first formulated by Vieillefosse [2], [3] and an approximate asymptotic solution was given therein. Cantwell [4] solved the restricted Euler equation analytically and made close comparisons between the solutions and DNS data bases for isotropic and homogeneously sheared turbulence with excellent agreement. However, when the restricted Euler equation is compared against DNS data bases for more complex turbulent flows the agreement is not as good, especially in regions of moderate to low dissipation (Cantwell [5]).

While the restricted Euler equation has already provided valuable insight into the velocity gradient geometry, there is still the need for further improvement, especially in complex flows. The objective of this paper is to improve upon the restricted Euler equation and extend its range of validity to cases more complex than isotropic turbulence. We first identify one possible reason for the inaccuracy of the restricted Euler equation in simulating flows with non-zero mean velocity gradients. It will be shown in Section 2 that the Reynolds-averaged restricted Euler equation violates the balance of mean momentum for virtually all homogeneous turbulent flows except isotropic and homogeneously sheared turbulence. It is for these two simple types of turbulence that the restricted Euler equation has yielded the best agreement (Ashurst et al [1], Cantwell [4]). After carefully demonstrating this shortcoming of the restricted Euler equation, we will then proceed, in Section 3, to develop a modified version of the restricted Euler equation which satisfies the mean momentum equation for general turbulent flows and, hence, is better capable of simulating more complex test cases. The modified restricted Euler equation is then tested in Section 4 and the implications for future research are discussed in the Conclusion.

\section{Theoretical Background}

The Euler equation in terms of the velocity gradients can be written in the form (Vieillefosse [2])

$$
\frac{d A_{i j}}{d t}+A_{i k} A_{k j}-\frac{1}{3} A_{m n} A_{n m} \delta_{i j}=H_{i j}
$$


where

$$
\begin{aligned}
A_{i j} & \equiv \frac{\partial U_{i}}{\partial x_{j}} \\
H_{i j} & \equiv-\left[\frac{\partial^{2} P}{\partial x_{i} \partial x_{j}}-\frac{1}{3} \frac{\partial^{2} P}{\partial x_{k} \partial x_{k}} \delta_{i j}\right] \\
\frac{d}{d t} & \equiv \frac{\partial}{\partial t}+U_{k} \frac{\partial}{\partial x_{k}}
\end{aligned}
$$

given that $U_{k}$ is the velocity vector and $P$ is the kinematic pressure. Vieillefosse [2] neglects the term $H_{i j}$ (the anisotropic pressure Hessian) leading to a simple dynamical system for the velocity gradients called the restricted Euler equation:

$$
\frac{d A_{i j}}{d t}+A_{i k} A_{k j}-\frac{1}{3} A_{m n} A_{n m} \delta_{i j}=0
$$

Vieillefosse [2] demonstrated that this model leads to a singularity (i.e., a divergence) in finite time of the velocity gradients. The geometry of the velocity gradients during the divergence obtained using approximate asymptotic methods revealed two important aspects of the velocity gradient tensor observed in DNS data: (i) the intermediate strain-rate eigenvector is positive and small; and (ii) the vorticity vector is aligned along the intermediate eigenvector.

Cantwell [4] obtained an elegant exact solution to the restricted Euler equation and made close comparisons with DNS data bases for isotropic and homogeneously sheared turbulence. The agreement was generally good. However, when the model is compared with mixing layer data the agreement is poor in regions of low dissipation (Cantwell [5]). Although the comparison can be improved with an ad hoc correction, questions about the validity of the model for more complex flows continue to persist.

We will now demonstrate that the restricted Euler equation leads to a violation of the balance of mean momentum for most non-trivial homogeneous turbulent flows. For this purpose, we decompose the instantaneous fields into mean and fluctuating parts as follows:

$$
\begin{aligned}
U_{i} & =\bar{U}_{i}+u_{i} \\
P & =\bar{P}+p \\
A_{i j} & =\bar{A}_{i j}+a_{i j} \\
& =\frac{\partial \bar{U}_{i}}{\partial x_{j}}+\frac{\partial u_{i}}{\partial x_{j}},
\end{aligned}
$$

where an overbar denotes an ensemble mean. The substitution of equation (4) into the restricted Euler equation (3), followed by an ensemble average, leads to the result

$$
\frac{\partial \bar{A}_{i j}}{\partial t}+\bar{U}_{k} \frac{\partial \bar{A}_{i j}}{\partial x_{k}}+\frac{\partial}{\partial x_{k}}\left(\overline{u_{k} a_{i j}}\right)+\bar{A}_{i k} \bar{A}_{k j}+\overline{a_{i k} a_{k j}}-\frac{1}{3}\left(\bar{A}_{m n} \bar{A}_{n m}+\overline{a_{m n} a_{n m}}\right) \delta_{i j}=0 .
$$


For incompressible homogeneous turbulence with constant mean velocity gradients, we have

$$
\begin{aligned}
& \frac{\partial \bar{A}_{i j}}{\partial t}=\frac{\partial \bar{A}_{i j}}{\partial x_{k}}=0, \\
& \frac{\partial}{\partial x_{k}}\left(\overline{u_{k} a_{i j}}\right)=0, \\
& \overline{a_{i k} a_{k j}}=\overline{\frac{\partial u_{i}}{\partial x_{k}} \frac{\partial u_{k}}{\partial x_{j}}}=\frac{\partial}{\partial x_{k}}\left(\overline{u_{i} \frac{\partial u_{k}}{\partial x_{j}}}\right)=0 .
\end{aligned}
$$

Hence, the mean momentum equation implied by the restricted Euler equation (3) reduces to

$$
\bar{A}_{i k} \bar{A}_{k j}-\frac{1}{3} \bar{A}_{m n} \bar{A}_{n m} \delta_{i j}=0
$$

Isotropic turbulence (for which $\bar{A}_{i j}=0$ ) and homogeneous shear flow (for which $\bar{A}_{i j}=S \delta_{i 1} \delta_{j 2}$, where $S$ is the shear rate) identically satisfy equation (7). However, virtually all other homogeneous turbulent flows violate the mean momentum equation (7) implied by the restricted Euler equation which is, therefore, inconsistent with the balance of mean momentum. For example, it is a simple matter to show that the following basic homogeneous turbulent flows violate equation (7):

Plane Strain

$$
\bar{A}_{i j}=\Gamma\left(\delta_{i 1} \delta_{j 1}-\delta_{i 2} \delta_{j 2}\right)
$$

Axisymmetric Expansion/Contraction

$$
\bar{A}_{i j}= \pm \frac{1}{2} \Gamma\left(\delta_{i 1} \delta_{j 1}+\delta_{i 2} \delta_{j 2}\right) \mp \Gamma \delta_{i 3} \delta_{j 3}
$$

Solid Body Rotation

$$
\bar{A}_{i j}=\omega\left(\delta_{i 1} \delta_{j 2}-\delta_{i 2} \delta_{j 1}\right)
$$

where $\Gamma$ is the strain rate and $\omega$ is the rotation rate. Of course, each of these basic flows satisfy the mean Euler equation since, for homogeneous turbulence, the Reynolds-averaged Navier-Stokes equation reduces to the Reynolds-averaged Euler equation (homogeneity causes the mean viscous terms to vanish). At the minimum, a viable model of turbulence must satisfy the balance of mean momentum; in fact, it has been argued that many Reynolds stress models owe the limited success that they have had exclusively to the fact that they are consistent with the mean balance laws. On these grounds, we conclude that the restricted Euler equation cannot serve as a viable model for the analysis of general homogeneous turbulent flows.

\section{Modified Restricted Euler Equation}

In this Section, we will formulate a modified restricted Euler equation that is consistent with any mean velocity gradient field. The incompressible Navier-Stokes equation for the instantaneous velocity field is given by

$$
\frac{\partial U_{i}}{\partial t}+U_{k} \frac{\partial U_{i}}{\partial x_{k}}=-\frac{\partial P}{\partial x_{i}}+\nu \frac{\partial^{2} U_{i}}{\partial x_{k} \partial x_{k}}
$$


where $\nu$ is the kinematic viscosity. By Reynolds averaging equation (11), the equation for the mean velocity field is obtained:

$$
\frac{\partial \bar{U}_{i}}{\partial t}+\bar{U}_{k} \frac{\partial \bar{U}_{i}}{\partial x_{k}}=-\frac{\partial \bar{P}}{\partial x_{i}}+\nu \frac{\partial^{2} \bar{U}_{i}}{\partial x_{k} \partial x_{k}}-\frac{\partial R_{i k}}{\partial x_{k}}
$$

where $R_{i k} \equiv \overline{u_{i} \bar{u}_{k}}$ is the Reynolds stress tensor (as alluded to earlier, for homogeneous turbulence the last two terms on the right-hand-side of equation (12) vanish, yielding the Euler equation in terms of the mean velocity and mean pressure). The equation for the fluctuating velocity field is obtained by subtracting equation (12) from equation (11):

$$
\frac{\partial u_{i}}{\partial t}+U_{k} \frac{\partial u_{i}}{\partial x_{k}}=-u_{k} \frac{\partial \bar{U}_{i}}{\partial x_{k}}+\frac{\partial R_{i k}}{\partial x_{k}}-\frac{\partial p}{\partial x_{i}}+\nu \frac{\partial^{2} u_{i}}{\partial x_{k} \partial x_{k}} .
$$

The fluctuating velocity gradient equation is then given by

$$
\frac{d a_{i j}}{d t}+a_{i k} a_{k j}=-a_{i k} \bar{A}_{k j}-\bar{A}_{i k} a_{k j}-u_{k} \frac{\partial \bar{A}_{i j}}{\partial x_{k}}+\frac{\partial^{2} R_{i k}}{\partial x_{k} \partial x_{j}}-\frac{\partial^{2} p}{\partial x_{i} \partial x_{j}}+\nu \frac{\partial^{2} a_{i k}}{\partial x_{k} \partial x_{k}} .
$$

By contracting equation (14), a Poisson equation for the fluctuating pressure field is obtained:

$$
\frac{\partial^{2} p}{\partial x_{k} \partial x_{k}}=-a_{l k} a_{k l}-2 a_{l k} \bar{A}_{k l}+\frac{\partial^{2} R_{l k}}{\partial x_{l} \partial x_{k}} .
$$

From the Poisson equation for pressure and equation (14) we get

$$
\begin{aligned}
\frac{d a_{i j}}{d t} & +a_{i k} a_{k j}+a_{i k} \bar{A}_{k j}+\bar{A}_{i k} a_{k j}+u_{k} \frac{\partial \bar{A}_{i j}}{\partial x_{k}} \\
& -\frac{\partial^{2} R_{i k}}{\partial x_{k} \partial x_{j}}-\frac{1}{3}\left[a_{l k} a_{k l}+2 a_{l k} \bar{A}_{k l}-\frac{\partial^{2} R_{l k}}{\partial x_{l} \partial x_{k}}\right] \delta_{i j}=h_{i j}
\end{aligned}
$$

where

$$
h_{i j}=-\left(\frac{\partial^{2} p}{\partial x_{i} \partial x_{j}}-\frac{1}{3} \frac{\partial^{2} p}{\partial x_{k} \partial x_{k}} \delta_{i j}\right)+\nu \frac{\partial^{2} a_{i j}}{\partial x_{k} \partial x_{k}} .
$$

If, in the spirit of the restricted Euler equation, we set $h_{i j}=0$ in equation (16), we obtain the general version of our modified restricted Euler equation for fluctuating velocity gradients. Equation (16) is not very useful for inhomogeneous mean flow fields since its solution requires knowledge of the fluctuating velocity field which is external to the dynamical system of equations. However, for homogeneous mean flows, the dynamical system of equations is closed in terms of the fluctuating velocity gradients: $\bar{A}_{i j}$ is a constant tensor and the second derivative of the Reynolds stress tensor vanishes, leading to the tractable form

$$
\frac{d a_{i j}}{d t}+a_{i k} a_{k j}+a_{i k} \bar{A}_{k j}+\bar{A}_{i k} a_{k j}-\frac{1}{3}\left(a_{l k} a_{k l}+a_{l k} \bar{A}_{k l}+\bar{A}_{l k} a_{k l}\right) \delta_{i j}=0 .
$$

This can be rewritten as

$$
\frac{d a_{i j}}{d t}+A_{i k} A_{k j}-\bar{A}_{i k} \bar{A}_{k j}-\frac{1}{3}\left(A_{l k} A_{k l}-\bar{A}_{l k} \bar{A}_{k l}\right) \delta_{i j}=0 .
$$


For homogeneous turbulent flows with constant mean velocity gradients we have

$$
\frac{d A_{i j}}{d t}=\frac{d a_{i j}}{d t}
$$

Hence, the modified restricted Euler equation for the total velocity gradient is given by

$$
\frac{d A_{i j}}{d t}+A_{i k} A_{k j}-\frac{1}{3} A_{l k} A_{k l} \delta_{i j}=\bar{A}_{i k} \bar{A}_{k j}-\frac{1}{3} \bar{A}_{l k} \bar{A}_{k l} \delta_{i j}
$$

A comparison of equations (3) and (21) reveals the difference between the original and the modified restricted Euler equation. The direct effect of the mean velocity gradient tensor on the total velocity gradient is retained intact by the present model. Recall that the original model has no explicit dependence on the mean velocity gradient tensor. The mean velocity gradient terms on the righthand-side of equation (21) survive since the mean pressure Hessian is not neglected - a feature that maintains consistency with the balance of mean momentum. It is a simple matter to show that the original restricted Euler equation for the velocity gradients is recovered for isotropic turbulence $\left(\bar{A}_{i j}=0\right)$ and homogeneous shear flow $\left(\bar{A}_{i j}=S \delta_{i 1} \delta_{j 2}\right)$. Due to the presence of the new terms, equation (21) does not appear to be as easily amenable to the type of analysis performed by Cantwell [4] on the original equation. Numerical solutions of (21) are also not straightforward due to the fact that the restricted Euler equation is known to lead to a singularity (divergence of the velocity gradients) in finite time (Vieillefosse [2], Cantwell [4]). Therefore, from this point onwards, we concern ourselves only with the numerical analysis of equation (18) for the fluctuating velocity gradients.

A viable analysis of the modified restricted Euler equation hinges on the use of a quantity that does not diverge in finite time, yet contains all of the important information about the geometry of the fluctuating velocity gradient tensor. The normalized fluctuating velocity gradient tensor $b_{i j}$, defined as

$$
b_{i j}=\frac{a_{i j}}{\sqrt{\varepsilon}}
$$

where $\varepsilon=a_{m n} a_{m n}$, contains all of the geometrical information about the fluctuating velocity gradient tensor and, by definition, is bounded in magnitude $\left(-1 \leq b_{i j} \leq 1\right)$. The evolution equation for $b_{i j}$ is easily obtained from equation (18):

$$
\begin{aligned}
\frac{d b_{i j}}{d t}= & -\sqrt{\varepsilon}\left[b_{i k} b_{k j}-\frac{1}{3} \delta_{i j} b_{l k} b_{k l}-b_{i j} b_{l m} b_{m n} b_{l n}\right] \\
& -\left[b_{i k} \bar{A}_{k j}+\bar{A}_{i k} b_{k j}-\frac{2}{3} \delta_{i j} b_{p q} \bar{A}_{q p}-b_{i j}\left(b_{p q} b_{p r} \bar{A}_{r q}+\bar{A}_{p q} b_{q r} b_{p r}\right)\right]
\end{aligned}
$$

This equation highlights some important aspects of the behavior of the velocity gradients in homogeneous turbulence. For fluid particles of high dissipation, characterized by

$$
\varepsilon \gg \bar{A}_{i j} \bar{A}_{i j}
$$


the first term on the right-hand-side (RHS) of equation (23) is the dominant one. This term is oblivious to the mean velocity gradients and is present in isotropic as well as anisotropic turbulent flows. As a result, in regions of high dissipation, we can expect a certain degree of universality in the behavior of the fluctuating velocity gradients. In fluid particles of low dissipation,

$$
\varepsilon \ll \bar{A}_{i j} \bar{A}_{i j}
$$

so that the second term on the RHS of equation (23) is more important. The mean velocity gradient imprints its signature on the fluctuating velocity gradients through this term. Hence, in areas of low dissipation, we expect the fluctuating velocity-gradient behavior to be different for different types of homogeneous mean flows.

Equation (23) is not yet suitable for numerical computations due to the presence of $\sqrt{\varepsilon}$ (which diverges in finite time) in the numerator of the first term on the right-hand-side. To make this equation more amenable to numerical computations, the evolution of $b_{i j}$ is considered in normalized time:

$$
\left.d t^{\prime}=\frac{d t}{\tau}, \quad \text { (where } \tau \equiv \frac{1}{\sqrt{\varepsilon}}\right)
$$

The quantity $\tau$ is the time scale inherent to a fluid particle. In areas of high dissipation, the time scale is small and vice-versa. The equation for $b_{i j}$ in normalized time is given by

$$
\begin{aligned}
\frac{d b_{i j}}{d t^{\prime}}= & -\left[b_{i k} b_{k j}-\frac{1}{3} \delta_{i j} b_{l k} b_{k l}-b_{i j} b_{l m} b_{m n} b_{l n}\right] \\
& -\tau\left[b_{i k} \bar{A}_{k j}+\bar{A}_{i k} b_{k j}-\frac{2}{3} \delta_{i j} b_{p q} \bar{A}_{q p}-b_{i j}\left(b_{p q} b_{p r} \bar{A}_{r q}+\bar{A}_{p q} b_{q r} b_{p r}\right)\right]
\end{aligned}
$$

If $\bar{A}_{i j}=0$ (as in the original restricted Euler equation), equation (27) can be solved in normalized time without any knowledge of $\tau$. When the mean velocity field is non-trivial, $\tau$ needs to be known to solve equation (23). The evolution of the time scale in normalized time is given by

$$
\frac{d \tau}{d t^{\prime}}=\tau b_{i j} b_{j k} b_{i k}+\tau^{2}\left[b_{i j} \bar{A}_{j k} b_{i k}+\bar{A}_{i j} b_{j k} b_{i k}\right]
$$

Equations (27) and (28) are well suited for numerical computations even if the unnormalized velocity gradients diverge in finite time.

\section{Results and Discussion}

In this Section, we will solve equations (27) and (28) numerically for isotropic and anisotropic homogeneous turbulent flows. We will show that the behavior of important fluctuating velocity gradient tensor invariants in the anisotropic cases are different from the isotropic case, highlighting the importance of the new terms in the modified restricted Euler equation. The anisotropy of the fluctuating velocity gradient tensor induced by the anisotropic mean flow will also be investigated. The modified restricted Euler equation appears to capture some known anisotropic behavior in 
rapidly distorted flows, as we will soon demonstrate. The velocity gradient tensor invariants selected for consideration are (Cantwell [4], [5])

$$
Q=-\frac{1}{2} a_{i j} a_{j i} \text { and } R=-\frac{1}{3} a_{i j} a_{j k} a_{k i}
$$

Cantwell [4] proves that the invariant $Q_{0}$ defined as

$$
Q_{0}^{3}(t)=\frac{27}{4} R^{2}(t)+Q^{3}(t)
$$

is constant in the case of the original restricted Euler equation. From the above definition, it is clear that $Q_{0}$ is the value of $Q$ when $R=0$.

While isotropic turbulence lends itself easily to analysis, it is of limited practical value. It is usually more reasonable to simplify flows of practical interest to more general anisotropic homogeneous turbulent flows. Comparisons of the modified restricted Euler equation with anisotropic turbulence DNS data would be quite useful. However, such a comparison is deferred to a more detailed future study. In this work we restrict our attention to another equally interesting question: how does the presence of mean velocity gradients alter the behavior of the fluctuating velocity gradients as predicted by the modified restricted Euler equation? There is evidence from DNS that the mean velocity gradient tensor has a significant effect on the fluctuating velocity gradient tensor. For example, Erlebacher and Sarkar [6] show that the fluctuating strain-rate eigenvectors exhibit preferential orientation with respect to the reference coordinates in their DNS of homogeneously sheared turbulence whereas in isotropic turbulence the eigenvectors have no preferential orientation. The original restricted Euler equation is oblivious to the mean velocity gradient field and hence is not generally capable of capturing this preferential orientation. The modified restricted Euler equation, on the other hand, is more sensitive to the mean velocity gradient field and, thus, has the capability of capturing this behavior. We will study the directional preference of the velocity gradient fluctuations by investigating the behavior of the anisotropic structure tensor function defined as

$$
F_{i j}=\overline{b_{k i} b_{k j}}-\frac{1}{3} \delta_{i j}
$$

and the anisotropic dissipation rate tensor defined as

$$
G_{i j}=\overline{b_{i k} b_{j k}}-\frac{1}{3} \delta_{i j}
$$

(It should be noted that in viscous flows, the anisotropic dissipation rate tensor, as a result of being normalized, has no explicit dependence on viscosity.) In isotropic turbulence, the components of these tensors are all zero. The magnitudes and signs of the non-zero components should therefore convey a good description of the anisotropic structure of the fluctuating velocity gradient tensor. Also to be investigated is the the behavior of the invariants $Q$ and $R$ and their normalized counterparts $q$ and $r$ in anisotropic flows. 
The results presented below are obtained as follows. An ensemble of 4096 fluid particles are considered. The initial velocity gradient values for each particle are assigned from a DNS velocity gradient field for isotropic turbulence (Yeung [7]). The initial velocity gradient field is normalized with the Kolmogorov length scale so that

$$
\overline{\varepsilon(0)} \equiv \overline{a_{i j} a_{i j}}(0)=1
$$

The time evolution of the velocity gradients for each particle is obtained numerically by integrating equations (27) and (28) using a predictor-corrector Runge-Kutta scheme. Physically, this is equivalent to suddenly subjecting an initially isotropic turbulence velocity gradient field to non-zero mean velocity gradients. With time, the fluctuating velocity gradients increase in magnitude. The components of the two anisotropic tensors of interest are calculated by ensemble averaging over all fluid particles.

The three types of mean velocity gradients used in the calculations are:

$$
\begin{aligned}
& \bar{A}_{i j}=S \delta_{i 1} \delta_{j 2} \quad \text { (homogeneous shear) } \\
& \bar{A}_{i j}=\Gamma \delta_{i 1} \delta_{j 1}-\Gamma \delta_{i 2} \delta_{j 2} \quad \text { (plane strain); } \\
& \bar{A}_{i j}=\omega \delta_{i 1} \delta_{j 2}-\omega \delta_{i 2} \delta_{j 1} \quad \text { (solid body rotation) }
\end{aligned}
$$

We assign the values of $S=\Gamma=\omega=10$. For each of the anisotropic cases, the ratio of the mean strain rate to the initial fluctuating strain rate is given by

$$
S \overline{\tau(0)}=\Gamma \overline{\tau(0)}=\omega \overline{\tau(0)}=10
$$

indicating that the flow is subjected to a somewhat rapid distortion. The implication is that, in equation (27), the terms involving the mean velocity gradients are initially dominant. With the passage of time, the strain ratio gets smaller and the non-linear terms in the fluctuating velocity gradient become more important.

Behavior of the invariants $Q$ and $R$. We compare the time evolution of the invariants $Q$ and $R$ following a sample fluid particle in the four cases. In each case, we calculate the time evolution of the two invariants starting from the same randomly chosen initial condition. The four resulting trajectories in the $Q-R$ phase space are provided in Figure 1. Since the initial velocity gradient tensor is identical in all of the four cases, the starting point of all the trajectories is the same. In the isotropic case, the trajectory is similar to that obtained in Cantwell [4], as it should be. The value of $R$ increases monotonically at all times. The $Q$ value shows an initial increase, but at large times it decreases monotonically. The trajectory of the homogeneous shear flow case is qualitatively similar. It is distinctly different in the early times, but asymptotes to the isotropic trajectory at later times. The plane-strain trajectory is quite different. The values of both $Q$ 
and $R$ increase monotonically. The trajectory of the rotating case is even more interesting. The trajectory loops around several times in the $Q-R$ phase space and no monotonic trend is observed initially. In the absence of any analytical solution of the velocity gradient equation (as in Cantwell [4]), we cannot make any precise statements about the effect of the mean velocity gradient on the invariants. However, one inference that can be drawn from the sample trajectories shown in Figure 1 is that the modified restricted Euler equation leads to very different behaviors of the invariants for different mean velocity gradients. The deviation from isotropic behavior is highest initially when the magnitudes of the velocity gradients are low - which in Eulerian coordinates would translate to regions of low dissipation. This suggests that the deviation from isotropic behavior observed in the low dissipation regions of the wake flow considered by Cantwell [5] could, perhaps, be due to the effect of the mean velocity gradients rather than due to the anisotropic part of the pressure Hessian or the viscous terms.

Behavior of $F_{i j}$. In order to understand the preferential orientation of the velocity gradient tensor with respect to the reference coordinates, we examine the behavior of the diagonal components of the anisotropic structure tensor function $F_{i j}$. Since the velocity gradients are initially isotropic, the components of $F_{i j}$ are initially zero for all three anisotropic cases.

In the homogeneous shear flow case (Figure 2a), the spanwise component of the anisotropic structure tensor $\left(F_{33}\right)$ is nearly unaffected by the shear (in the 1,2-plane). The streamwisecomponent $\left(F_{11}\right)$ decreases from zero, rapidly in the beginning, and, at later times, asymptotes to a value of approximately -0.2 . This indicates that the fluctuating velocity gradients in the streamwise direction are milder than in the other directions, and, hence, the length scale in this direction is likely to be larger. The cross-stream component $\left(F_{22}\right)$ increases rapidly at first and, then, asymptotes to a positive value of approximately 0.2 . This implies that the velocity field length scale is smallest in the cross-stream direction.

In the plane strain case (Figure 3a), the gradients in the extensive mean flow direction seem to smooth out very rapidly initially leading to $F_{11}$ becoming negative. After reaching its minimum value of about $-0.25, F_{11}$ increases somewhat, asymptoting to a value close to -0.15 . The spanwise component $\left(F_{33}\right)$ is somewhat affected. It peaks at a value of about 0.08 and asymptotes to a slightly lower value. The component in the compressive mean flow direction $\left(F_{22}\right)$ increases very rapidly in the beginning. This indicates that the effect of mean flow compression is to steepen the fluctuating velocity gradients. After peaking at a value close to $0.2, F_{22}$ then decreases gradually.

Rapid solid body rotation of an initially isotropic velocity field does not appear to affect the isotropy of the structure tensor function immediately (Figure 4a). Notice that all three components stay close to zero initially for a short duration. This behavior is consistent with the observations of Speziale, Mansour and Rogallo [8] in their numerical study of the evolution of an initially 
isotropic velocity field subjected to a rapid solid body rotation. They found that rapid rotation produces inertial waves that survive for several eddy turnover times leaving the velocity field with approximately isotropic one-point statistics. Once these inertial waves are damped, anisotropy due to mean rotation can set in. At later times, the axial component, $F_{33}$, starts to decrease (in comparison with $F_{11}$ and $\left.F_{22}\right)$ gradually and monotonically. This could indicate that the gradients in the axial direction are getting smaller and smaller consistent with the Taylor-Proudman theorem, which states that, at infinite rotation rates, the gradients in the axial direction should go to zero. The components $F_{11}$ and $F_{22}$ display identical behavior, implying that the velocity gradients are statistically symmetric in the plane of rotation.

It should be cautioned that the behavior of the structure tensor function depicted here is likely to be very close to physical reality only for early times. At later times, when the fluctuating velocity gradients get very large, the neglected $h_{i j}$ terms are likely to play key a role, which, can perhaps, cause the behavior of $F_{i j}$ to deviate from that described above.

Behavior of $G_{i j}$. Often in turbulence modeling, the dissipation is taken to be isotropic. In anisotropic flows, the degree of departure of the dissipation tensor from isotropy is not well understood. Here, we examine the anisotropic part the dissipation tensor, $G_{i j}$, calculated from the modified restricted Euler equation. We again present results for only the diagonal components for the sake of brevity.

For the case of homogeneous shear flow (Figure $2 \mathrm{~b}$ ), $G_{11}$ increases monotonically. The growth is rapid at first, and $G_{11}$ ultimately asymptotes to a value close to 0.18 . This implies that the velocity fluctuations in the streamwise direction are dissipated more rapidly than those in the other directions. The spanwise component, $G_{33}$, remains nearly unaffected. The cross-stream component, $G_{22}$, decreases monotonically to offset the increase in $G_{11}$. An interesting point to note is that the behavior of $G_{i j}$ is exactly opposite to that of $F_{i j}$.

In the plane strain case (Figure $3 \mathrm{~b}$ ), the components of $G_{i j}$ and $F_{i j}$ have identical evolutions. The dissipation in the extensive direction is substantially lower than in the compressive and spanwise directions. On the other hand, solid body rotation suppresses dissipation in the axial direction (Figure 4b). The dissipation along both directions in the plane of the rotation exhibit identical behavior at higher dissipation levels.

The above figures clearly indicate that the dissipation tensor is quite anisotropic when the mean flow gradients are strong. Although the results shown here may not be completely valid at later times, they are quite credible for short elapsed times where the rapid distortion limit formally applies. 


\section{Conclusion}

In this paper, we have clearly demonstrated that the original restricted Euler equation is not consistent with the balance of mean momentum for most homogeneous turbulent flows with non-zero mean velocity gradients. An alternate model for the fluctuating velocity gradients in homogeneous turbulent flows - which we refer to as the modified restricted Euler equation - was developed in equations (16) and (18). The new model was derived by making a simplification that is similar in spirit to the one made in the original restricted Euler equation (Vieillefosse [2]) - this time applied to the fluctuating pressure field instead. Unlike the original restricted Euler model, the modified model for the total velocity gradient is sensitive to the type of the mean velocity gradients to which the flow is subjected. Analytical solutions, of the type obtained by Cantwell [4] for the original restricted Euler equation, were not attempted due to the additional complexity introduced by the new terms in the modified equation. The modified equation, like the original restricted Euler equation, leads to a singularity (i.e., divergence) of the fluctuating velocity gradients in finite time. A strategy for capturing the geometry of the fluctuating velocity gradient tensor during this divergence was developed in equations (27) and (28). Calculations performed using the modified restricted Euler equation reveal important differences between the fluctuating velocity gradients in isotropic and anisotropic turbulence. Some known anisotropic features of the fluctuating velocity gradients (including a possible Taylor-Proudman reorganization in flows with a strong solid body rotation) appear to be captured by the new model. These results for anisotropic homogeneous turbulence, combined with the previously demonstrated ability of the original restricted Euler equation to capture many of the features of isotropic turbulence, leads us to believe that the new modified restricted Euler equation can serve as a valuable dynamical model for the analysis of the geometry of the velocity gradient tensor in homogeneous turbulent flows. 


\section{References}

[1] W. T. Ashurst, A. R. Kerstein, R. M. Kerr and C. H. Gibson, Alignment of vorticity and scalar gradient with strain rate in simulated Navier-Stokes turbulence. Phys. Fluids A 30 , 2243 (1987).

[2] P. Vieillefosse, Local interaction between vorticity and shear in a perfect incompressible fluid. J. Phys. (Paris) 43, 837 (1982).

[3] P. Vieillefosse, Internal motion of a small element of fluid in an inviscid flow. Physica A 125, 150 (1984).

[4] B. J. Cantwell, Exact solution of a restricted Euler equation for the velocity gradient tensor. Phys. Fluids A 4, 782 (1992).

[5] B. J. Cantwell, On the behavior of velocity gradient tensor invariants in direct numerical simulations of turbulence. Phys. Fluids A 5, 2008 (1993).

[6] G. Erlebacher and S. Sarkar, Statistical analysis of the rate of strain tensor in compressible homogeneous turbulence. Phys. Fluids A 5, 3240 (1993).

[7] P.K. Yeung. Private communication.

[8] C. G. Speziale, N. N. Mansour and R. S. Rogallo, The decay of isotropic turbulence in a rapidly rotating frame. Proceedings of the 1987 Summer Program of the Center for Turbulence Research, Stanford University, p. 205 (1987). 


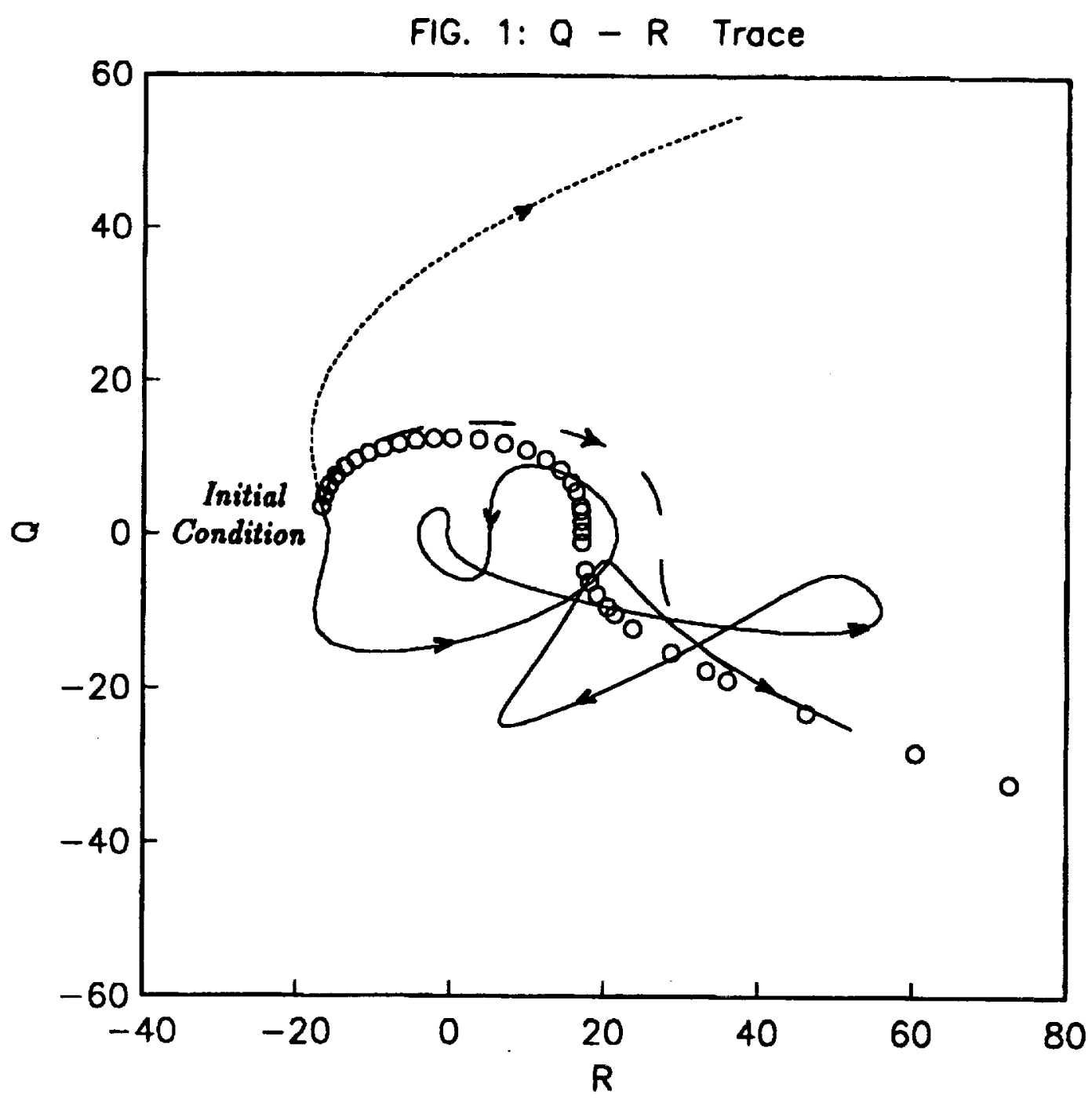

Figure 1: Trajectory in $Q-R$ phase space of fluid particles for various mean velocity fields: (O) isotropic; - - homogeneous shear; -. - plane strain; and, - solid body rotation. 


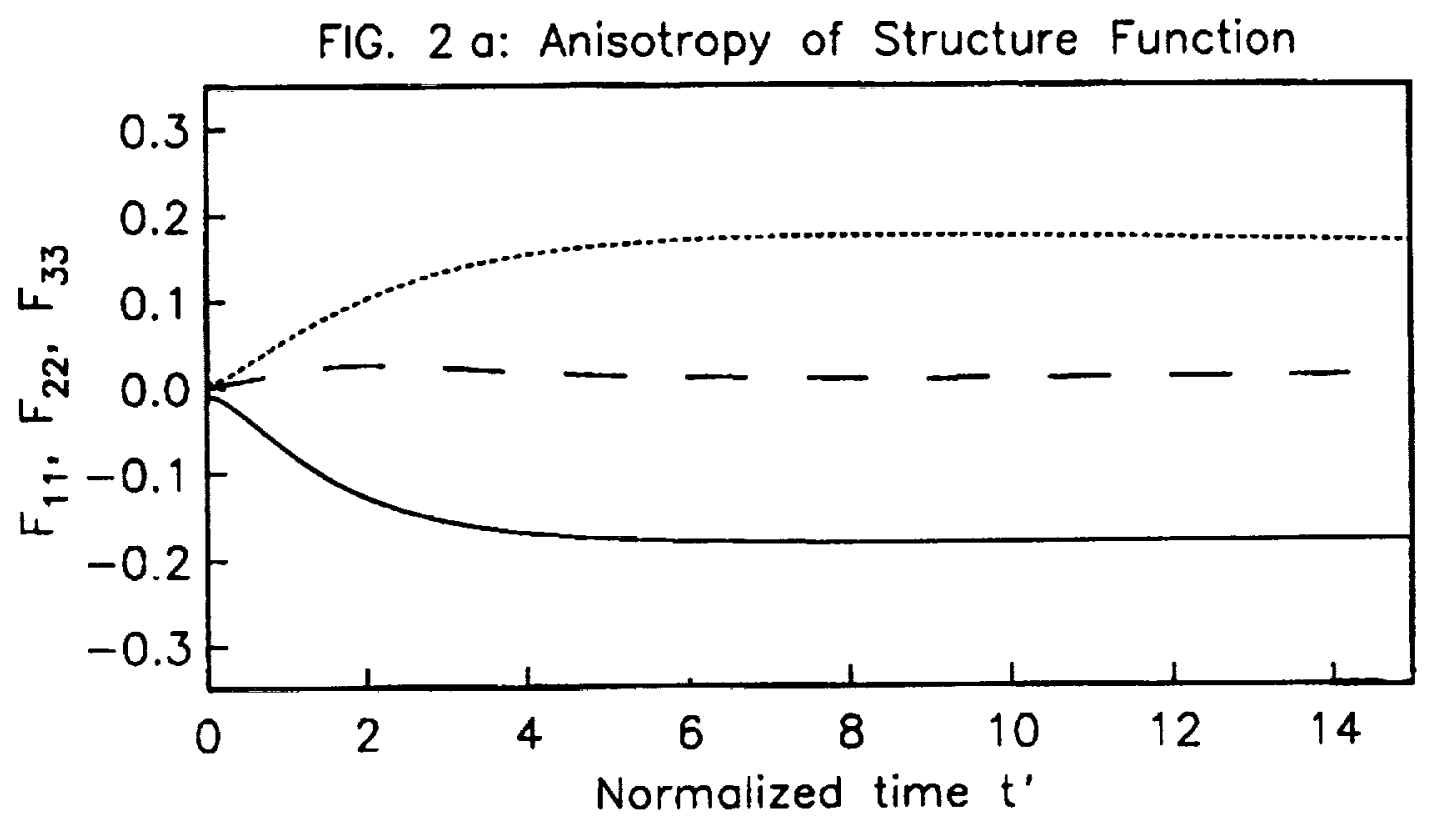

FIG. 2 b: Anisotropy of Dissipation

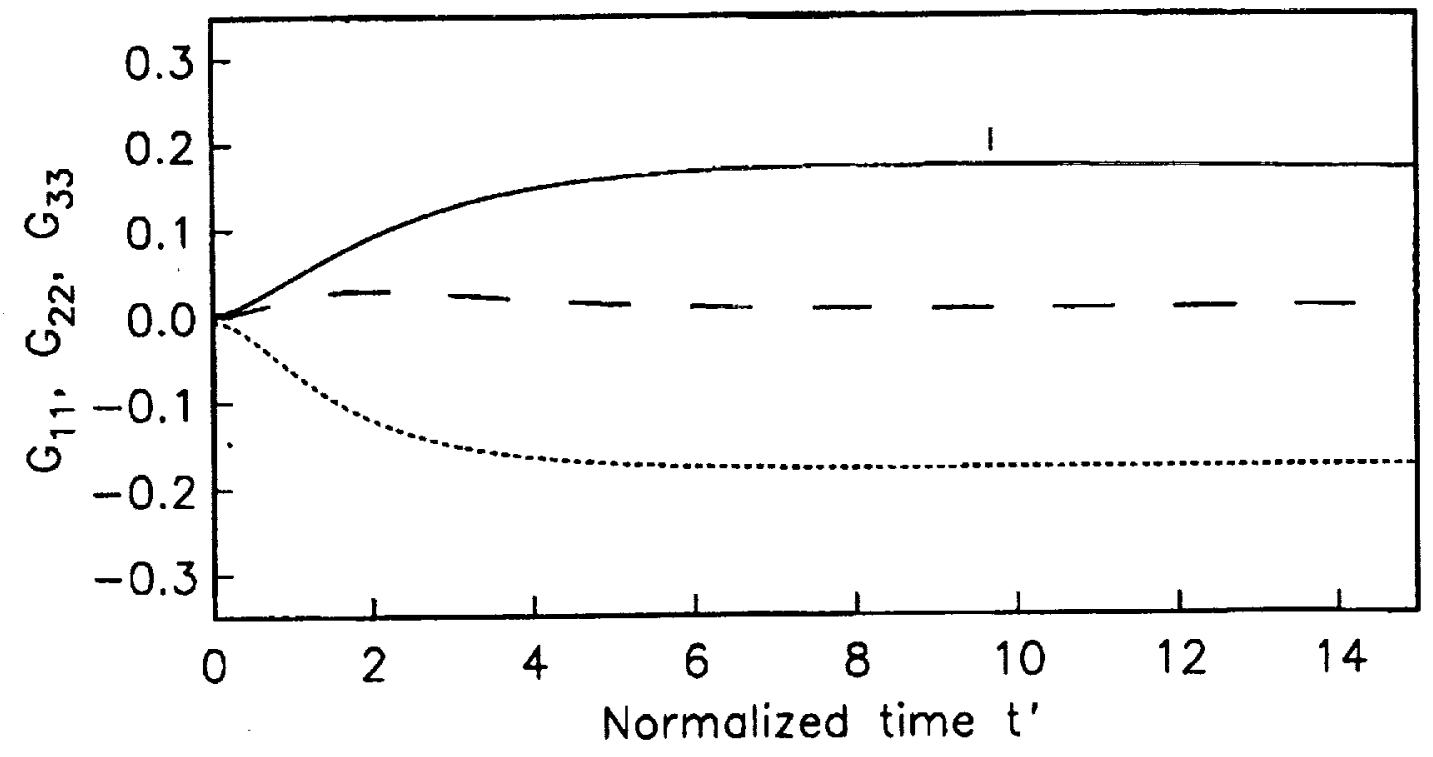

Figure 2: Time evolution of $F_{i j}$ and $G_{i j}$ in homogeneous shear flow: (a) $-F_{11}, \ldots F_{22}$, and $--F_{33} ;$ (b) $-G_{11}, \ldots G_{22}$, and $--G_{33}$. 


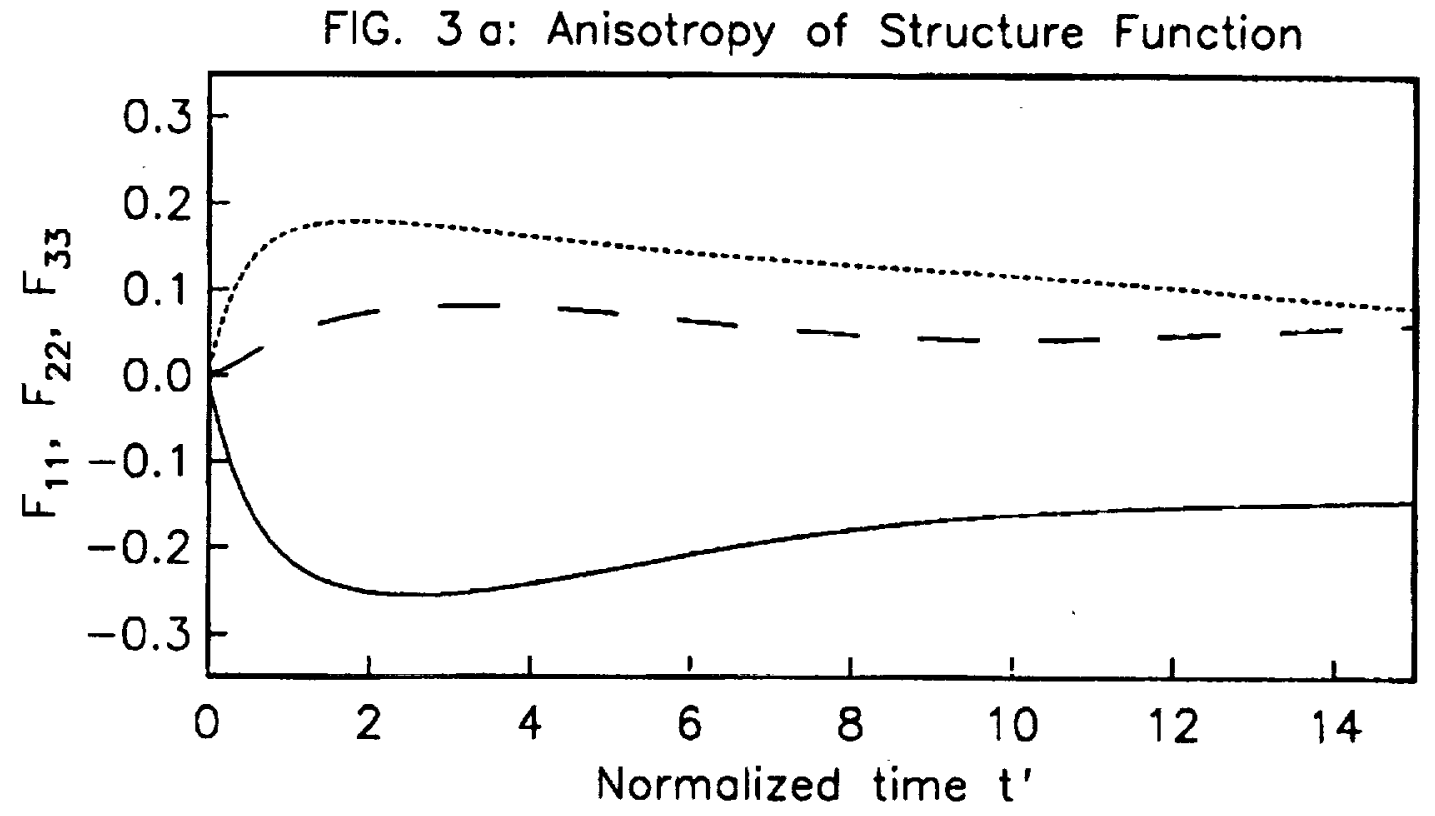

FIG. 3 b: Anisotropy of Dissipation

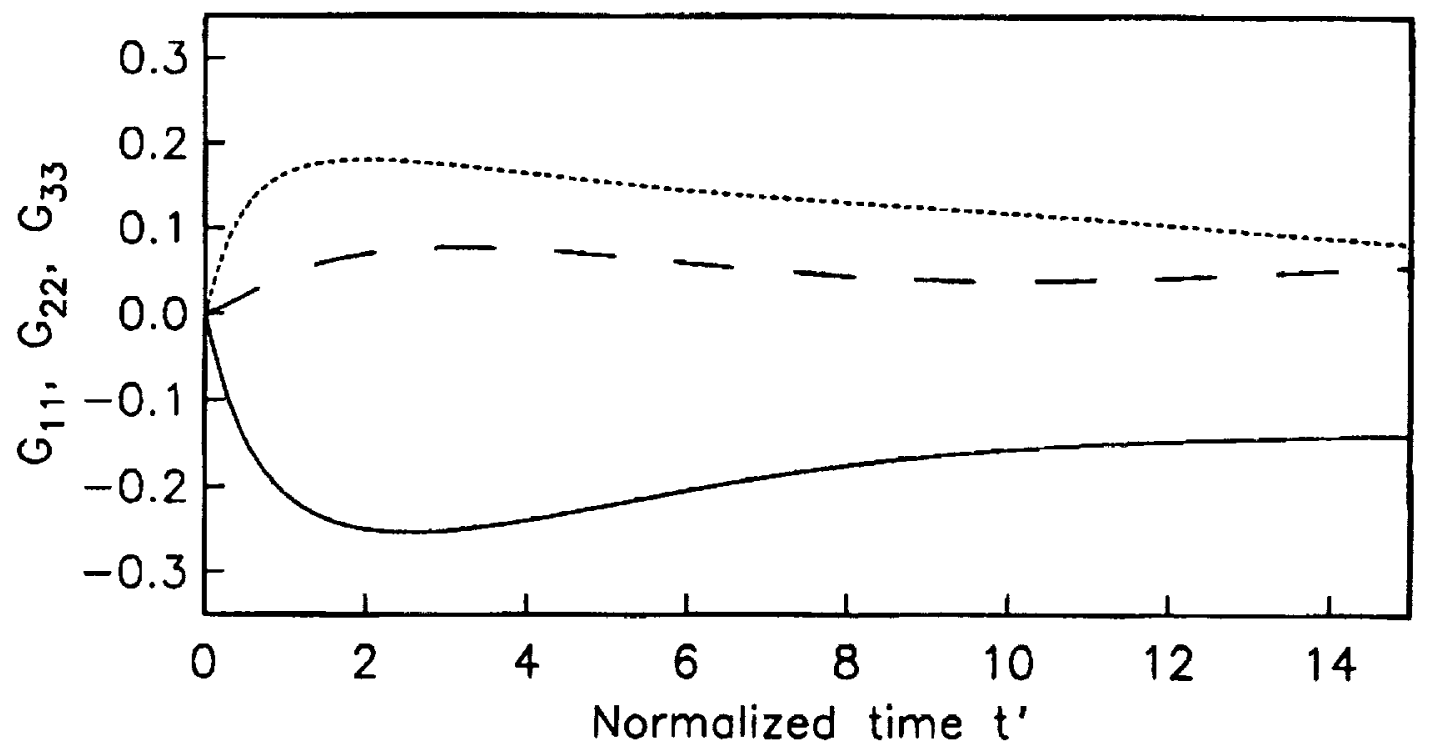

Figure 3: Time evolution of $F_{i j}$ and $G_{i j}$ in the plane strain case. Legend the same as in Figure 2. 
FIG. 4 a: Anisotropy of Structure Function

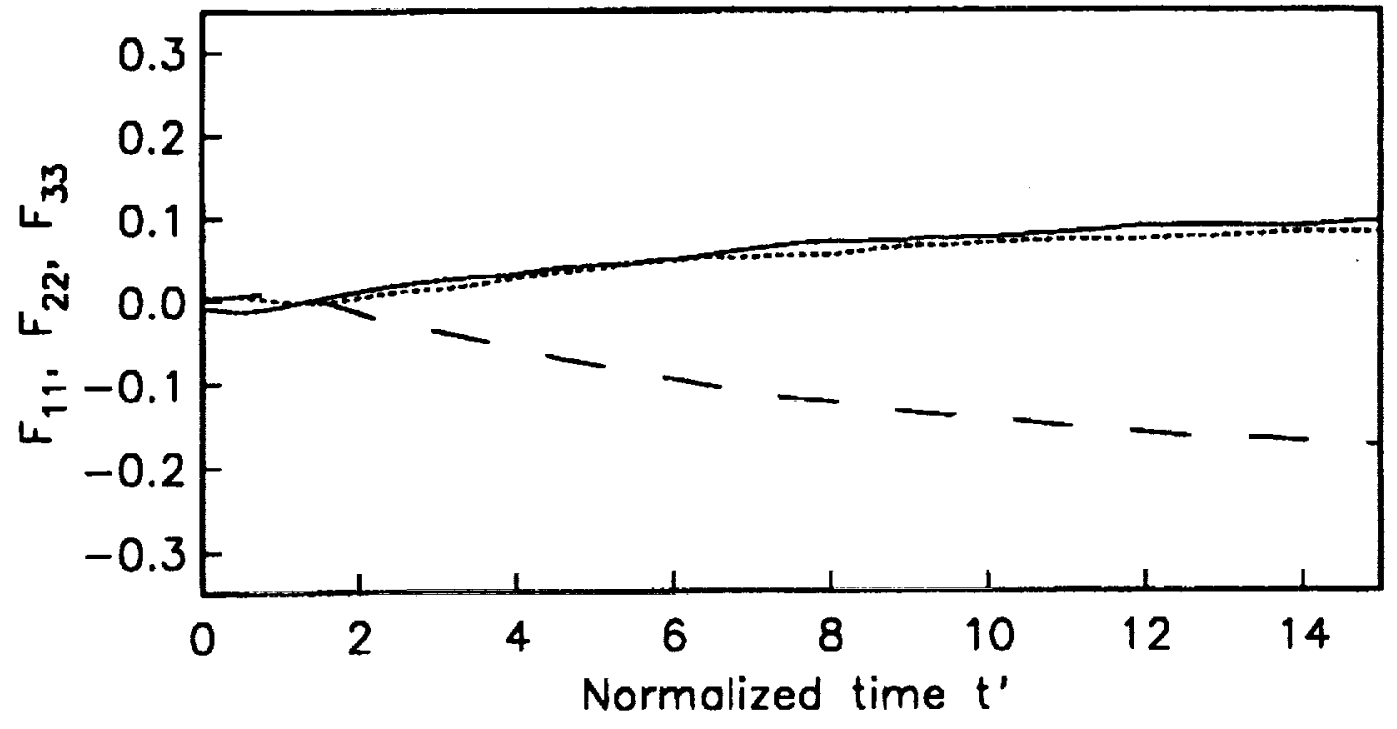

FIG. 4 b: Anisotropy of Dissipation

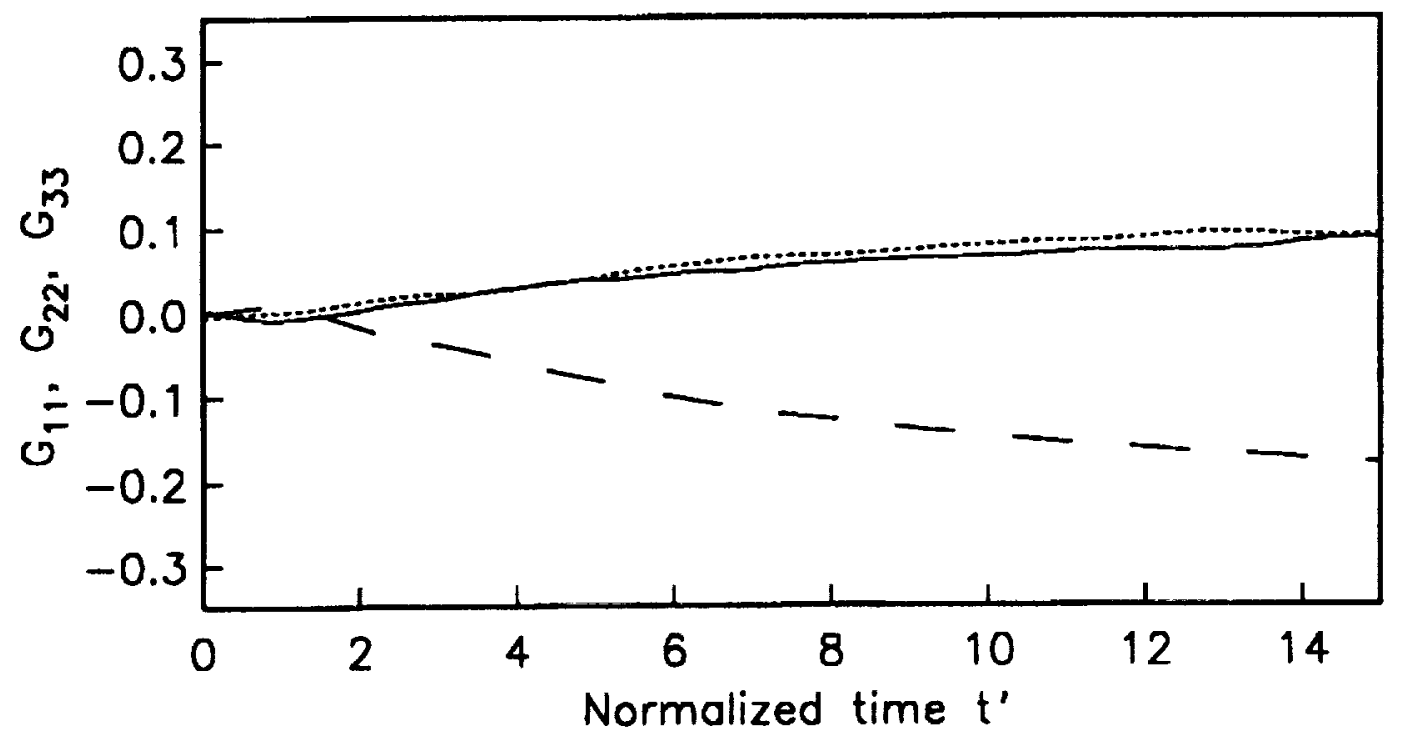

Figure 4: Time evolution of $F_{i j}$ and $G_{i j}$ in the solid body rotation case. Legend the same as given in Figure 2. 


\begin{tabular}{|c|c|c|c|}
\hline \multicolumn{3}{|c|}{ REPORT DOCUMENTATION PAGE } & $\begin{array}{l}\text { Form Approved } \\
\text { OMB No. 0704-0188 }\end{array}$ \\
\hline \multicolumn{4}{|c|}{ 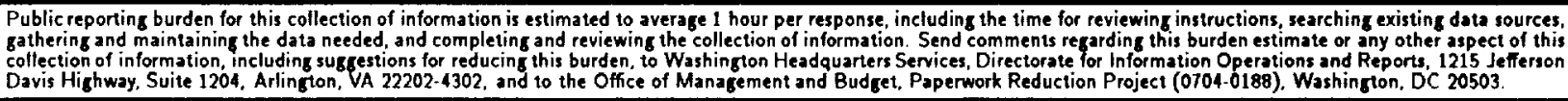 } \\
\hline 1. AGENCY USE ONLY(Leave blank) & $\begin{array}{l}\text { 2. REPORT DATE } \\
\text { September } 1994\end{array}$ & $\begin{array}{l}\text { 3. REPOR } \\
\text { Contra }\end{array}$ & $\begin{array}{l}\text { ND DATES COVERED } \\
\text { ort }\end{array}$ \\
\hline \multicolumn{3}{|c|}{$\begin{array}{l}\text { 4. TITLE AND SUBTITLE } \\
\text { A MODIFIED RESTRICTED EULER EQUATION FOR TURBU- } \\
\text { LENT FLOWS WITH MEAN VELOCITY GRADIENTS }\end{array}$} & $\begin{array}{l}\text { 5. FUNDING NUMBERS } \\
\text { C NAS1-19480 } \\
\text { WU } 505-90-52-01\end{array}$ \\
\hline \multicolumn{4}{|l|}{$\begin{array}{l}\text { 6. AUTHOR(S) } \\
\text { Sharath S. Girimaji } \\
\text { Charles G. Speziale }\end{array}$} \\
\hline \multicolumn{3}{|c|}{$\begin{array}{l}\text { 7. PERFORMING ORGANIZATION NAME(S) AND ADDRES5(ES) } \\
\text { Institute for Computer Applications in Science } \\
\text { and Engineering } \\
\text { Mail Stop 132C, NASA Langley Research Center } \\
\text { Hampton, VA 23681-0001 }\end{array}$} & $\begin{array}{l}\text { 8. PERFORMING ORGANIZATION } \\
\text { REPORT NUMBER } \\
\text { ICASE Report No. } 94-76\end{array}$ \\
\hline \multicolumn{3}{|c|}{$\begin{array}{l}\text { 9. SPONSORING/MONITORING AGENCY NAME(S) AND ADDRESS(ES) } \\
\text { National Aeronautics and Space Administration } \\
\text { Langley Research Center } \\
\text { Hampton, VA 23681-0001 }\end{array}$} & $\begin{array}{l}\text { 10. SPONSORING/MONITORING } \\
\text { AGENCY REPORT NUMBER } \\
\text { NASA CR-194979 } \\
\text { ICASE Report No. } 94-76\end{array}$ \\
\hline \multicolumn{4}{|c|}{$\begin{array}{l}\text { 11. SUPPLEMENTARY NOTES } \\
\text { Langley Technical Monitor: Michael F. Card } \\
\text { Final Report } \\
\text { Submitted to Physics of Fluids }\end{array}$} \\
\hline \multicolumn{3}{|c|}{$\begin{array}{l}\text { 12a. DISTRIBUTION/AVAILABILITY STATEMENT } \\
\text { Unclassified-Unlimited } \\
\text { Subject Category } 34\end{array}$} & 12b. DISTRIBUTION CODE \\
\hline \multicolumn{4}{|c|}{$\begin{array}{l}\text { 13. ABSTRACT (Maximum } 200 \text { words) } \\
\text { The restricted Euler equation captures many important features of the behavior of the velocity gradient tensor } \\
\text { observed in direct numerical simulations (DNS) of isotropic turbulence. However, in slightly more complex flows } \\
\text { the agreement is not good, especially in regions of low dissipation. In this paper, it is demonstrated that the } \\
\text { Reynolds-averaged restricted Euler equation violates the balance of mean momentum for virtually all homogeneous } \\
\text { turbulent flows with only two major exceptions: isotropic and homogeneously-sheared turbulence. A new model } \\
\text { equation which overcomes this shortcoming and is more widely applicable is suggested. This model is derived from } \\
\text { the Navier-Stokes equation with a restricted Euler type approximation made on the fluctuating velocity gradient } \\
\text { field. Analytical solutions of the proposed modified restricted Euler equation appear to be difficult to obtain. } \\
\text { Hence, a strategy for numerically calculating the velocity gradient tensor is developed. Preliminary calculations } \\
\text { tend to indicate that the modified restricted Euler equation captures many important aspects of the behavior of the } \\
\text { fluctuating velocity gradients in anisotropic homogeneous turbulence. }\end{array}$} \\
\hline
\end{tabular}


